\title{
Adaptive Smart Power Control Algorithm for LTE Downlink Cross-Tier Interference Avoidance
}

\author{
Indoor Power Constraint for LTE Downlink Cross-Tier Interference Avoidance
}

\author{
Yen-Wei Kuo ${ }^{1}, \mathrm{Li}_{-}$Der Chou ${ }^{1}$ and Yi-Ming Chen ${ }^{2}$ \\ ${ }^{1}$ Department of Computer Science and Information Engineering \\ ${ }^{2}$ Department of Information Management \\ National Central University \\ Jhongli Dist., Taoyuan City, 32001 Taiwan \\ 11E-mail:965402015@cc.ncu.edu.tw and cld@csie.ncu.edu.tw \\ ${ }^{2}$ E-mail: cym@cc.ncu.edu.tw
}

\begin{abstract}
In the deployment of heterogeneous Long Term Evolution (LTE), due to the unmanaged power range of femtocells, inter-cell interference problems cause severe degradation in network performance, particularly in Closed Subscriber Group (CSG) mode. In the paper, we proposed an effective approach termed Adaptive Smart Power Control Algorithm (ASPCA), which can be applied to cluster users in coverage hole and to tackle cross-tier interference issues by determining an appropriate serving range for femtocells without requiring neither standard changing nor complicated negotiation among cells. In simulation model, several frequency-reuse-based schemes have been investigated. As a result, we demonstrate that compared to conventional way, the ASPCA significantly improved the macrocell performance about $51.32 \%$ in average user throughput but less affected the femtocell performance around $8.63 \%$ reduction in average user throughput and caused a negligible $0.46 \%$ overall loss in average throughput. The ASPCA also provided well conditions for frequency-reuse-based schemes of Inter-Cell Interference Coordination (ICIC), such as Soft Frequency Reuse (SFR) and Fractional Frequency Reuse (FFR), which can be integrated to provide the most benefit for cellular networks.
\end{abstract}

Keywords-LTE; macrocell; femtocell; interference; CSG; ICIC

\section{INTRODUCTION}

Nowadays, the Heterogeneous Network (HetNet) has been became the most important scenario. The HetNet enables the interoperability between various multi-coverage protocols and devices such as macrocells, picocells, and femtocells that provide different coverages and capacities for different wireless application scenarios such as outdoor environment and indoor building. Some researches indicate that more than $70 \%$ of data traffic originates from indoors [1] [2] where the small cells, such as femtocells, are necessary to be deployed for achieving a high spectral efficiency [3]. Due to shorter transmission distances, the smaller cell size enables a more efficient spatial reuse of spectrum and higher channel gains [4].

The femtocell is an effective way to enhance the coverage and capacity for indoor environment. It is a small and lowpower base station, also referred to as HeNB, usually deployed in indoor area to recover coverage holes where users cannot receive any signal in from the wireless network. The femtocell not only recovers the coverage holes, but also improves the capacity of cellular network by operating through backhaul connection to broadband network, bring in a lot of new services without a significant increase in network deployment and management cost, such as CAPital EXpenditures (CAPEX) and OPerational EXpenditures (OPEX).

The main benefit of femtocell is offloading the network traffic from LTE macrocells. Therefore femtocell is usually deployed in the overlapped area of macrocell and shared the same spectrum with the macrocell, thus leading to the wellknown co-channel interference problem. Moreover, due to the nature of residential deployment, the femtocells are preferred to operate in restricted access mode in which only those users belonging to the same Closed Subscriber Group (CSG) [5] can be allowed to access. In such case, the CSG creates severe interference especially to the macrocell users closed to the femtocells without having access permission. In contrast to the macrocell, the femtocell users are less affected by the macrocell since they are usually in the vicinity of serving cell [6]. Although, even recent studies addressed that the cochannel femtocells less affects the macrocell performance, but users could lose the opportunity to get satisfactory data service [7].

To meet the goal of spectrum efficiency, inter-cell interference management is important task in LTE system. Several interference management technologies have been proposed in 3GPP standardization [5], in which Inter-Cell Interference Coordination (ICIC) is considered as the most promising approach for inter-cell interference mitigation [8]. The ICIC is achieved by applying restrictions of radio sources or transmit power, and additional signaling over X2 interface for information exchange between neighboring cells is needed; So, the ICIC can be roughly grouped into two categories named frequency reuse and power control [9]. But the signaling overhead for information exchange via X2 interface is highly affected by the number of coordinated cells [10]. Moreover, the $\mathrm{X} 2$ interface is not available between the femtocells and macrocells for now [6] [10].

In addition, the ICIC schemes can be classified into two types: proactive schemes and reactive schemes. The proactive schemes are prevention strategies in advance, such as Fractional Frequency Reuse (FFR) and Soft Frequency Reuse (SFR), and the reactive schemes are compensation strategies in 
afterward, such as High Interference Indication (HII), Relative Narrowband Transmit Power Indicator (RNTP), and Overload Indicator $(\mathrm{OI})$. Both kinds of strategies must be complemented with each other in order to effectively overcome the inter-cell interference.

The radio resources restriction can be implemented though frequency reuse schemes such as Fractional Frequency Reuse (FFR) and Soft Frequency Reuse (SFR), which can significantly improve cell-edge performance [11]. In the schemes, the whole system bandwidth is divided into several partitions, and then the inter-cell interferences are mitigated by increasing the spatial distance between neighboring cells. The main difference between SFR and FFR is that the FFR allocates only a part of frequency resources for users instead of all available frequency resources. However, since pre-planned frequency-time allocation is needed for frequency reuse schemes, these schemes cannot deal with rapid and dynamic spatial-temporal variation in cellular networks [11].

The power control is an alternative approach of ICIC. Since the interference experienced by cells and User Equipments (UEs) depend upon the neighbor cell's transmit power, the power restriction schemes can be used for mitigating the intercell interference. The power control provides the flexibility to allocate different power levels on different Resource Blocks (RBs) instead of constant spectral density of transmit power over the whole system bandwidth. For instance, the Smart Power Control Algorithm (SPCA) proposed in [12] provides a limited coverage to alleviate inter-cell interference by Reference Signal Received Power (RSRP) measurement in idle and connected states, but there is, though, no further description regarding the determination of cell specific parameters. The femtocells can adjust its maximum transmit power according to the following formula:

$$
\begin{gathered}
P_{t x}=\max \left\{\operatorname { m i n } \left[\alpha\left(C R S E+10 \log \left(N_{r b}^{d l} N_{s c}^{r b}\right)\right)\right.\right. \\
\left.\left.+\beta, P_{\text {max }}\right], P_{\min }\right\}
\end{gathered}
$$

where the $N_{R B}^{D L}$ represents the number of resource blocks within downlink system bandwidth. The $N_{S C}^{R B}$ denotes the number of Sub-Carriers (SC) within a resource block. $P_{\max }$ and $P_{\min }$ are maximum power and minimum power allowed by the transmission, respectively. A resource block consists of 12 subcarriers with $15 \mathrm{kHz}$ sub-carrier spacing. The CRSE in $\mathrm{dBm}$, also called RSRP, is the average liner power of Reference Signals (RS) transmitted from the strongest co-channel interfering cell and measured by the UEs [13-14]. The cell specific parameters $\alpha$ and $\beta$ are used for altering power range of serving cell. The value of $\alpha$ is usually chosen to provide sufficient margin for providing better mobile user experience by manufacturer, causing additional interference to other users. Consequently, the users of the neighboring cell experienced a strong interfering signal leakage from the nearby femtocells. Even if the interference is reduced by power control, but the useful signal strength is reduced as well [3]. Therefore, an appropriate coverage range should be determined adaptively.

The enhanced Inter-Cell Interference Coordination (eICIC) a time-domain ICIC proposed by 3GPP provides time-sharing means for downlink transmissions [15]. The Almost Blank Sub-frames (ABS) is one of eICIC approach defined in Release
10. For the purpose of interference avoidance, ABS bitmaps are used to informing the neighboring cells to avoid transmitting in non-ABS subframes; ideally, the ABS would be totally blank subframes. The ABS bitmap should be configured depending on cell's loading and the number of trapped cell's users. However, due to the absence of X2 interface between the femtocells and macrocells, the LTE systems tends to configure the same ABS bitmap for all femtocells, this leads a low data efficiency [16].

In the paper, we concentrate on dealing with the downlink cross-tier interference between the macrocells and femtocells by employing the power restriction scheme based on SPCA, not only the use's interference, but also users' Quality of Service (QoS) requirements are taken into account. The proposed Adaptively Smart Power Control Algorithm (ASPCA) can adaptively determine the range of coverage hold, and then set the cell-specific parameters to an appropriate value in order to control cells' coverage range without requiring neither standard changing nor complicated negotiation as well as signaling consumption among the cells. By separating cellcenter users and cell-edge users and using adaptive power control, the ASPCA can be combined with frequency reuse schemes to benefit cell's performance. As for multi-room problem, it can be regarded as an extension of the single room problem by leveraging wall penetration among cells in the same way.

The rest of the paper is organized as follows. Section II describes the proposed mechanism. Section III proposes simulation model. Section IV presents the simulation results and discussions about the mechanism we presented. Section V concludes this paper.

\section{THE PROPOSED MECHANISMS}

In this section, we describe the detail of the proposed mechanism including indoor-outdoor user clustering algorithm, Adaptive Power Control Algorithm (APCA), and the SPCA. In the proposed mechanism, UEs are equipped with a simplified Down Link (DL) receiver circuitry used to determine the RSRP) from interfering-cells [4] to aware the quality of Resource Block (RB) UEs experienced. Figure 3 depicts the flowchart of the proposed mechanism used in femtocells for the heterogeneous scenario. In the proposed approach, we attempt to find the range of femtocell's coverage hold by RSRP measurement, and then to determine an appropriate transmission power for femtocells. 


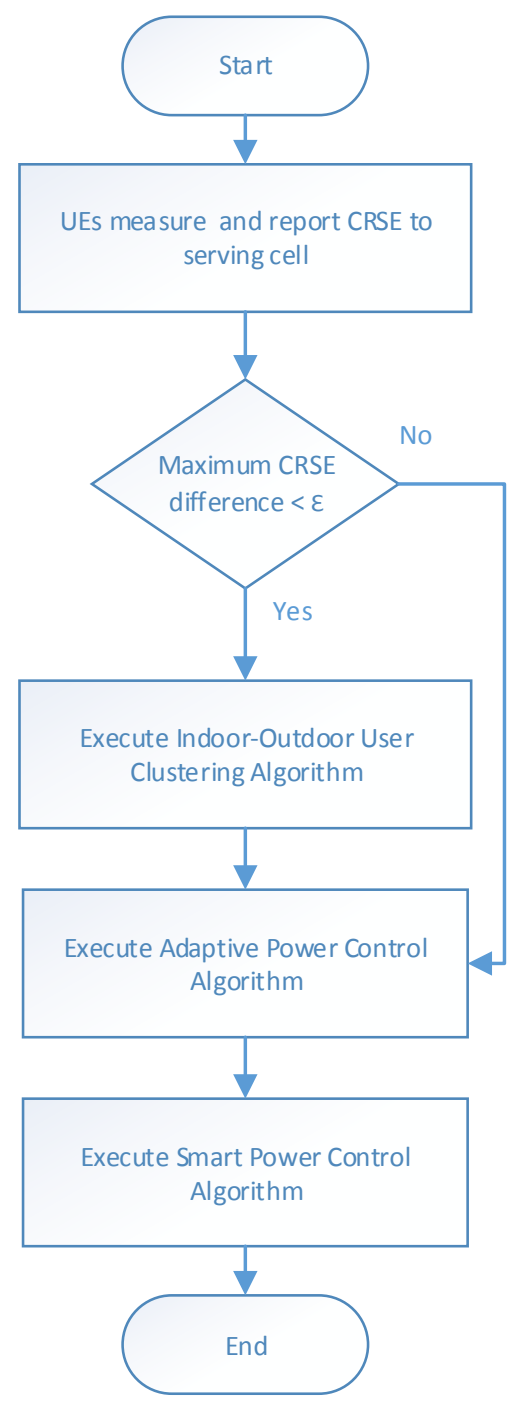

Figure 1. The flowchart of the Adaptive Smart Power Control Algorithm

In the flowchart, firstly, UEs measure and report CRSE of the strongest co-channel interference to its serving cell. If the maximum CRSE difference between serving cell and UEs greater than a threshold $\varepsilon$, the indoor-outdoor UEs clustering algorithm does not need to be performed. This entails that there are currently no interfering signals or no outdoor users. Otherwise, the serving cell perform the clustering algorithm in order to group indoor users. Next, the adaptive power control algorithm calculates the cell specific parameter $\alpha$ according to the maximum CRSE received by the indoor users. Finally, the serving cell sets its transmission power to meet SINR target by the smart power control algorithm. The further description of proposed components as follows:

\section{A. Indoor-Outdoor Users Clustering Algorithm}

The indoor-outdoor users clustering algorithm is used to separate femtocell's users into indoor and outdoor clusters based on wall penetration loss between them. The clustering algorithm is summarized by the following procedure as shown in Figure 2.

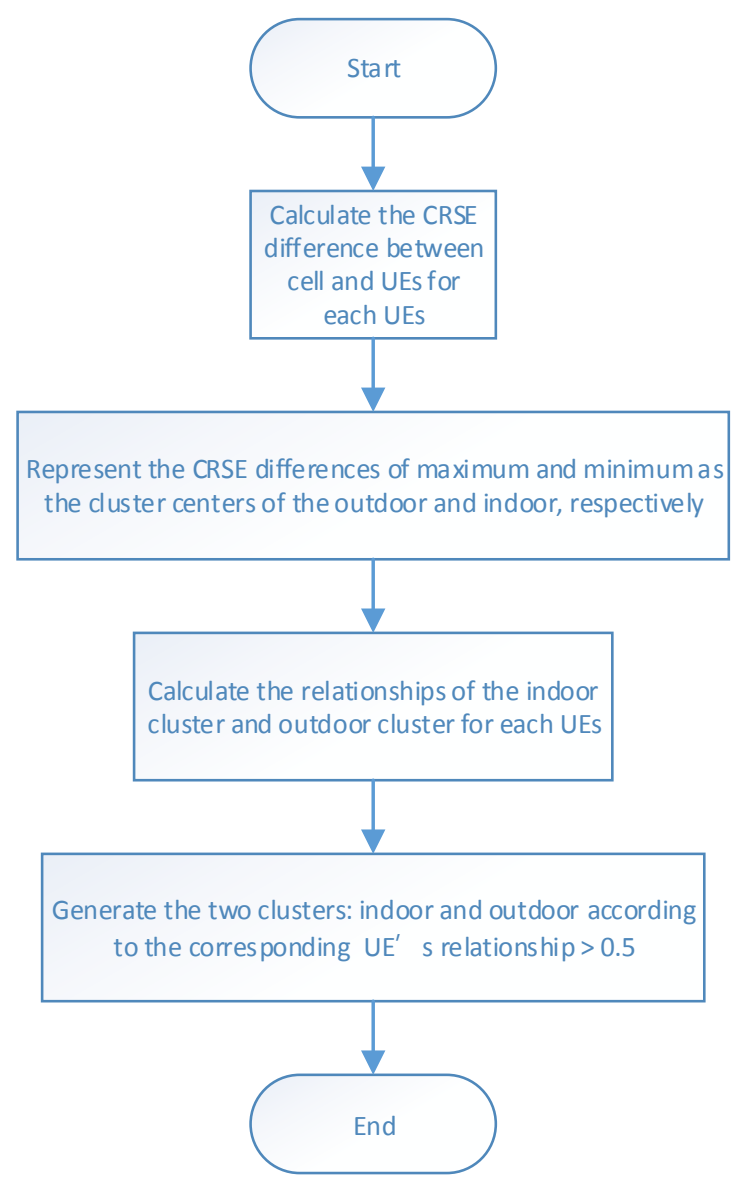

Figure 2. The flowchart of the Indoor-Outdoor UEs Clustering Algorithm

To simplify computation complexity, we calculate the CRSE difference between cell and UEs since that femtocells are usually deployed in the indoor environment. Finally, UEs are grouped in the indoor cluster or in the outdoor cluster according to the corresponding relationships to cluster centers which are presented as the minimum and maximum CRSE differences, respectively. The relationship functions are given as

$$
\begin{gathered}
\mu_{\text {indoor }}(k)=\frac{1}{1+\frac{\left|C R S E_{k}-C R S E_{\min }\right|}{\left|C R S E_{k}-C R S E_{\max }\right|}} \\
\mu_{\text {outdoor }}(k)=\frac{1}{1+\frac{\left|C R S E_{k}-C R S E_{\max }\right|}{\left|C R S E_{k}-C R S E_{\min }\right|}} \\
\mu_{\text {outdoor }}(k)+\mu_{\text {indoor }}(k)=1
\end{gathered}
$$

where $C R S E_{k}$ is the CRSE difference between $k$ th UE and serving femtocell. $C R S E_{\max }$ and $C R S E_{\min }$ are the maximum and minimum of CRSE differences, respectively. In addition, the $\mu_{\text {indoor }}(k)$ and $\mu_{\text {outdoor }}(k)$ are complementary relationships.

\section{B. Adaptive Power Control Algorithm}

In this section, an adaptive power control algorithm based on the concept of Shannon channel capacity is introduced to 
adjust cell's downlink transmit power. A simple expression of Shannon Channel Capacity as shown in (3), which presents a number of data that can be transferred over a given communication channel with white Gaussian noise [17].

$$
\begin{aligned}
C & =B W \cdot \log _{2}\left(1+\frac{S}{N}\right) \\
& =B W \cdot \log _{2}\left(1+\frac{E_{b} R}{N_{o} B W}\right)
\end{aligned}
$$

where $C$ is channel capacity in unit of bits per second, $B W$ is the bandwidth available for communication, $S$ denotes the desirable signal power, and $N$ represents the power of white noise impair to the received signal. Moreover, the desirable signal and received noise can be further expressed as $S=E_{b} R$ and $N=N_{o} B W$, respectively. The $R$ denotes data rate in bits per second, $E_{b}$ represents the energy on a certain bit, and $N_{o}$ is the noise power per Hz. In practice, the channel capacity is more complicated for LTE system since a lot of factors should to be taken into consideration, such as interference signal, data rate, Modulation and Coding Schemes (MCS), size of IFFT/FFT, number of subcarriers (SCs), and cyclic prefix length, etc.

Considering the effect of interfering signal from neighbor cells, the expression can be reformulated as (4) to present the channel capacity on a resource block. The resource block is the smallest entity that can be scheduled for users in the frequency domain.

$$
\begin{aligned}
& C_{r b}=\Delta f_{r b} \log _{2}\left(1+\frac{\frac{E_{r b}}{\log _{2} M} R}{\left(\frac{I_{r b}}{\Delta_{r b}}+N_{o}\right) \Delta f_{r b}}\right) \\
& =\Delta f_{r b} \log _{2}\left(1+\frac{E_{r b}}{\left(\frac{I_{r b}}{\Delta f_{r b}}+N_{o}\right)} \frac{R_{r b}}{\Delta f_{r b}}\right)
\end{aligned}
$$

where the $E_{b}$ equals to $\frac{E_{r b}}{\log _{2} M}, R_{r b}$ equals to $\frac{R}{\log _{2} M}$, and $B W$ is $\Delta f_{r b}$ which equals to $180 \mathrm{kHz}$. In Orthogonal FrequencyDivision Multiplexing (OFDM) system, a resource block consists of 12 Sub-Carriers (SC) with spacing of $15 \mathrm{kHz}$. The $M$ and $R_{r b}$ denote adopted modulation type and data rate of a resource block, respectively, with respect to MCS selection. The $E_{r b}, I_{r b}$, and $N_{o}$ represent received energy, interfering signal, and noise signal presented on the resource block, respectively.

Because data rate can never exceed the channel capacity limit, the inequality (5) can be reformulated to provide a lower bound for acquiring the energy of a resource block $E_{r b}$ by defining the bandwidth utilization $\gamma$ as $\frac{R_{r b}}{\Delta f_{r b}}$; in OFDM system, the $R_{r b}$ equals to $N_{s c}^{r b} N_{s y m}^{s f} \log _{2} M$ kbps. The $N_{s c}^{r b}$ denotes the number of sub-carriers per resource block, and $N_{\text {sym }}^{s f}$ denotes the number of symbols in a $1 \mathrm{~ms}$ sub-frame; a sub-frame equals 1 TTI.

$$
\begin{aligned}
& \frac{R_{r b}}{\Delta f_{r b}} \leq \log _{2}\left(1+\frac{E_{r b}}{\left(\frac{I_{r b}}{\Delta f_{r b}}+N_{o}\right)} \frac{R_{r b}}{\Delta f_{r b}}\right) \\
& \gamma \leq \log _{2}\left(1+\frac{E_{r b}}{\left(\frac{I_{r b}}{\Delta f_{r b}}+N_{o}\right)} \gamma\right) \\
& \frac{E_{r b}}{\left(\frac{I_{r b}}{\Delta f_{r b}}+N_{o}\right)} \gamma \geq 2^{\gamma}-1 \\
& \frac{E_{r b}}{\left(\frac{I_{r b}}{\Delta f_{r b}}+N_{o}\right)} \geq \frac{2^{\gamma}-1}{\gamma} \\
& E_{r b} \geq \frac{2^{\gamma}-1}{\gamma}\left(\frac{I_{r b}}{\Delta f_{r b}}+N_{o}\right)
\end{aligned}
$$

Because a resource block consists of 12 subcarriers in LTE system, the energy on a subcarrier can be calculated as

$$
E_{s c}=E_{r b}-10 \log \left(N_{s c}^{r b}\right)
$$

where the $N_{s c}^{r b}$ equals to 12 . By setting the user satisfaction of data rate $R_{r b}$, the cell specific parameter $\alpha$ used in SPCA can be evaluate as:

$$
\begin{gathered}
\alpha=\frac{E_{s c}+10 \log \left(N_{r b}^{d l} N_{s c}^{r b}\right)}{C R S E+10 \log \left(N_{r b}^{d l} N_{s c}^{r b}\right)} \\
=\frac{E_{r e}}{C R S E}
\end{gathered}
$$

Furthermore, in our approach, the cell specific parameter $\beta$ is used to compensate path loss between UE and its serving cell. UEs can measure RSRP transmitted from serving-cell and compare it against the cell broadcast value in System Information Block2 (SIB Type2) on Physical Broadcast Channel $(\mathrm{PBCH})$ to evaluate the downlink path loss. Here, we assume that the estimated downlink path loss accurately reflects the uplink path loss.

\section{SYSTEM SIMULATION MODEL}

In contrast to other simulation mechanisms proposed in recently research [18] [19], we proposed a non-autonomous approach to reflect the effect of CSG mode, in which each user is belonged to a certain cell instead of associating with the cell with relative high signal strength. For simulation in dense urban, we consider a hexagonal of two-tier cellular network layout with an intentionally short of $100 \mathrm{~m}$ inter-cell distance. The simulation mode comprise an outdoor macrocell deployed in the center of Region of Interest (ROI) and six surrounding femtocells located in a $20 \times 20 \mathrm{~m}^{2}$ room, respectively. The femtocell's antenna is created in the middle of each building with only one floor, and a modified single-wall model [20] is used as propagation model for urban environment as:

$$
\begin{aligned}
P L_{\text {outdoor }}=40 & \left(1-4 \mathrm{e}^{-3} \text { Dhb }\right) \log _{10}(R) \\
& -10 \log _{10}(D h b) \\
& +21 \log _{10}(f)+80 d B
\end{aligned}
$$




$$
\begin{aligned}
P L_{\text {indoor }}=40(1 & \left.-4 \mathrm{e}^{-3} D h b\right) \log _{10}(R) \\
& -10 \log _{10}(D h b) \\
& +21 \log _{10}(f)+97 d B
\end{aligned}
$$

where $R$ is the distance from cell to the user in kilometers, $f$ is the carrier frequency in $\mathrm{MHz}$, and $D h b$ is the antenna height of cell in meters. In our simulation, we assumed $D h b$ equals to 15 meters.

The simulation configurations are given in Table I. At the beginning of simulation, the transmit power of femtocells are $20 \mathrm{dBm}$ while the transmit power of macrocell is $46 \mathrm{dBm}$. we considered three kinds of scenarios: no coordination, handoff,

\begin{tabular}{|c|c|}
\hline Network layout & Hexagonal/2-tier \\
\hline Network size & $200 \times 200 \mathrm{~m}^{2}$ \\
\hline Inter-cell distance & $100 \mathrm{~m}$ \\
\hline Map resolution & $1 \mathrm{~m}$ \\
\hline Indoor area & $20 \times 20 \mathrm{~m}^{2}$ \\
\hline Bandwidth & $20 \mathrm{MHz}$ \\
\hline Frame structure & Type 1/FDD \\
\hline Cyclic prefix & Normal cyclic prefix \\
\hline Transmission scheme & SISO \\
\hline Antenna type & $\begin{array}{l}\text { Omnidirectional with } 0 \mathrm{~dB} \\
\text { gain }\end{array}$ \\
\hline Noise density & $-174 \mathrm{dBm} / \mathrm{Hz}$ \\
\hline Propagation model & TS 36.942 \\
\hline Channel fading & Rayleigh \\
\hline Wall penetration loss & $17 \mathrm{~dB}$ \\
\hline Number of Macrocell & 1 \\
\hline Number of Femtocell & 6 \\
\hline Transmit power of Macrocell & $46 \mathrm{dBm}$ \\
\hline $\begin{array}{l}\text { Maximum transmit power of } \\
\text { Femtocell }\end{array}$ & $20 \mathrm{dBm}$ \\
\hline $\begin{array}{l}\text { Minimum transmit power of } \\
\text { Femtocell }\end{array}$ & $-10 \mathrm{dBm}$ \\
\hline Number of Macrocell users & 200 \\
\hline Number of users per Femtocell & 20 \\
\hline $\begin{array}{c}\text { Proportion of outdoor users per } \\
\text { Femtocell }\end{array}$ & 0.3 \\
\hline CRS $\widehat{E}_{U E}$ threshold, CRS $\widehat{E}_{\text {thres }}$ & $-50 \mathrm{dBm}$ \\
\hline
\end{tabular}
and frequency reuse in our simulations.

TABLE I SIMULATION CONFIGURATIONS.

First, the no-coordinated scenario is a benchmark used to present the conventional case of a heterogeneous network. Second, the handoff scenario consists of two strategies: the proposed approach and the handoff approach, used in indoors and outdoors respectively. Finally, the frequency reuse scenario is used for reporting the performance of ASPCA combined with frequency reuse scheme. In addition, because the performance of the proposed approach is highly affected by the user positions, the same setting of user position is used for all of simulation scenarios.
For the purpose of comparison, the Round-Robin (RR) scheduling is used as a resource allocation strategy for all simulation scenarios. RR can be seen as the fairest scheduling, in which users take turns to use shared resources. Although RR scheduling will lead to lower overall system performance since it does not take the channel conditions into account in the scheduling process, but it can be used to dramatically distinguish the cell capacity in different approaches. In fact, the scheduling strategy is implementation-specific and not part of 3GPP specifications.

\section{PERformance Results AND Discussions}

The comparison of adopting the ASPCA and conventional approach is illustrated in Figure 3, where the SINR gap of macrocell between the ASPCA and conventional method in the near building is about $24.36 \mathrm{~dB}$, and the SINR improvement in the cell center is about $20.86 \mathrm{~dB}$. This entails that the proposed approach can significantly improve communication quality for macrocell users. In practice, the communication quality of the macrocell has a crucial impact on system performance because the macrocell serves a significantly higher number of users than a femtocell.
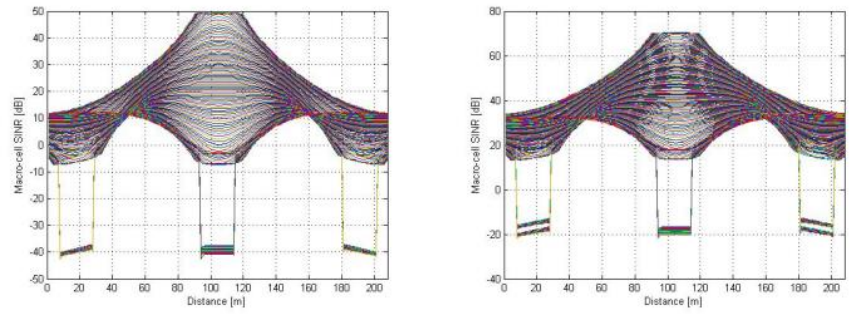

Figure 3. The SINR cumulative distribution of UEs.

Since the resource block is the smallest entity that can be scheduled for users in the frequency domain, the average throughput per resource block, as shown in Figure 4 and Figure 5 , is used to present the data rate variations without the effect of resource allocation strategy. Figure 4 compares the average throughput per resource block of the proposed approach against the conventional approach without any strategy of resource allocation for users.

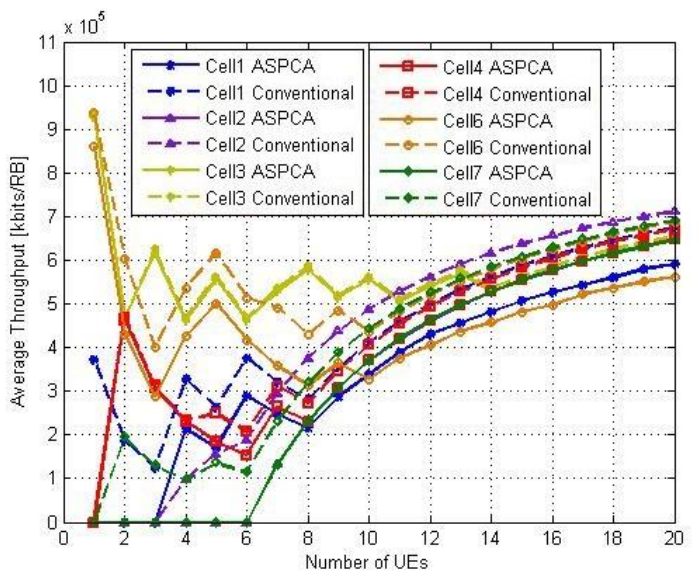

Figure 4. The comparison of average throughput per resource block of femtocells. 
In Figure 5, the throughput vibrations appear on the lowusers case because the users are randomly deployed in the ROI either outdoors or indoors. Although the proposed approach degraded average throughput per resource block by $8.63 \%$ in femtocells, but it also increased average throughput per resource block by $51.32 \%$ in macrocells, this intuitively leads to a $0.46 \%$ overall loss in average throughput depending on the number of femtocells in a macrocell. The overall loss mainly comes from the proportion of outdoor users per femtocell, this entails that a well-planned strategy for outdoor uses is very important to improve the system performance.

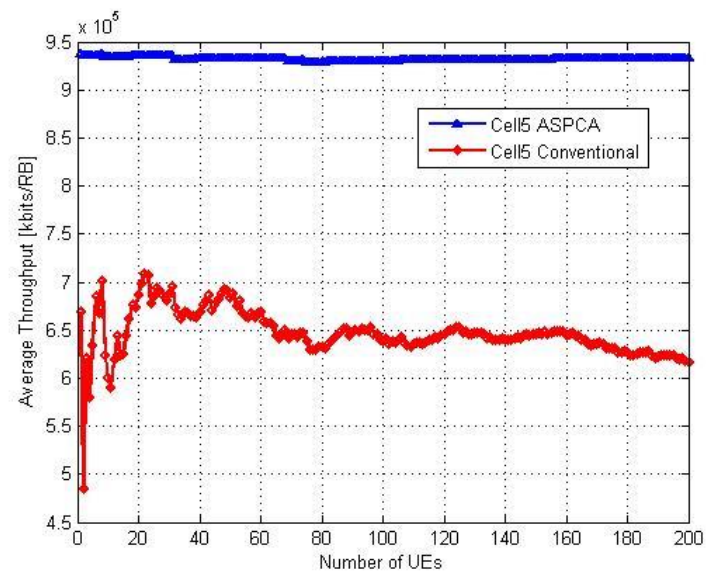

Figure 5. The comparison of average throughput per resource block of macrocells.

As we expected, Figure 6 depicts the proposed approach with handoff strategy offers superior performance as good as the SFR strategy because all users have a good SINR and all spectrum resources are fully exploited by the users. However, the ASPCA approach with FFR strategy reached only about a half of performance compared to the approach with SFR strategy due to underutilized spectrum resources. Intuitively, since the wider bandwidth can be used to accommodate more users, frequency-reuse-based schemes should adaptively take the number of outdoor users and network loading into account to determine the best resource partitioning. However, the proposed approach provides a similar performance compared to the convention approach in femtocells, but it offers superior performance as good as the handoff approach and SFR strategy in macrocells.

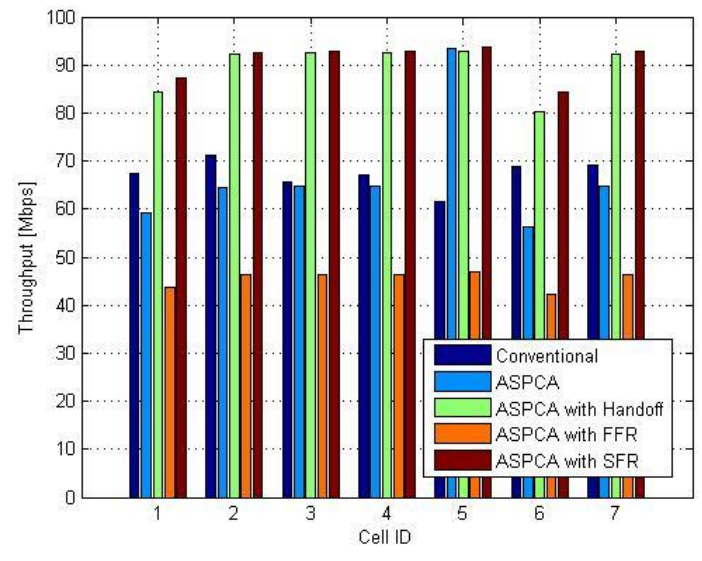

Figure 6 . The throughput of cells

The outage probability of cells is illustrated in Figure 7. In addition to macrocells, the outage probabilities of femtocells are equal to 0.3 , which is consistent with the proportion of outdoor users per femtocell.

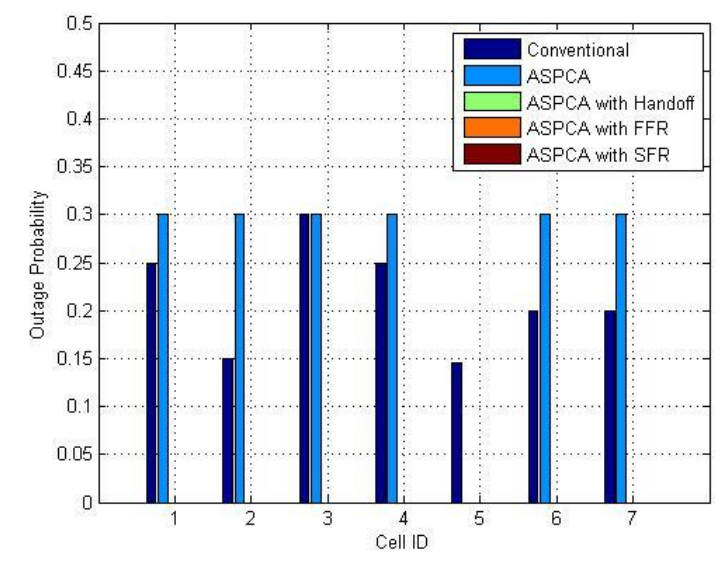

Figure 7. The outage probability of cells.

From the outage probability aspects, there are not many differences between the conventional approach and proposed approach in femtocells because the outdoor users are also impacted by macrocells. Furthermore, similar to the ASPCA approach with handoff strategy, the outage probability of the proposed approach with SFR and FFR is very low because all femtocells can ideally maintain a good channel quality for serving outdoor users.

In practice, the throughput in LTE system is fairly lower than the results from our simulation due to neglecting the overhead related to control signaling, such as PDCCH and PBCH channels, synchronization signals, and coding rates, and even a fully loaded network is assumed in the paper, but cells may experience a reduced load in practice. 


\section{CONCLUSIONS}

In the paper, we proposed an effective approach called Adaptive Smart Power Control Algorithm (ASPCA) to appropriately decide cell specific parameter for SPCA, which was specified in 3GPP specification. Due to the proper serving range, the ASPCA provided good conditions for frequency reuse schemes such as FFR and SFR used in femtocells. The simulation results show that ASPCA with handover strategy can offer a significant benefit for the network, which entails that the serving range of femtocells should be appropriately decided according to its coverage hold size in order to achieve the cross-tier interference avoidance. Furthermore, it should be note that the number of outdoor users and network loading should be adaptively taken into account in the frequency-reusebased schemes. Through simulations we illustrated that our proposed approach compared to the conventional way offers $51.32 \%$ increase in average user throughput of macrocell and a slight reduction about $8.36 \%$ in average user throughput of femtocells.

\section{REFERENCES}

[1] G. Mansfield, "Femtocells in the US market-business drivers and consumer propositions." FemtoCells Europe , pp. 1927-1948, 2008.

[2] S. Y. Wang, W. S. Guo, and T. O'Farrell, "Low energy indoor network: deployment optimization," EURASIP Journal on Wireless Communications and Networking, vol. 2012.1, pp. 1-15, 2012.

[3] K. R. Jacobson and W. A. Krzymien, "Multihop relaying and multiple antenna techniques: performance trade-offs in cellular systems," EURASIP Journal on Wireless Communications and Networking, vol. 2011.1, pp 1-19. 2011.

[4] Z. Bharucha, G. Auer, T. Abe, and N. Miki, "Femto-to-Macro Control Channel Interference Mitigation via Cell ID Manipulation in LTE," IEEE Vehicular Technology Conference (VTC Fall), pp. 1-6, 2011.

[5] 3GPP, TSG TS 36.300, "Evolved Universal Terrestrial Radio Access (EUTRA) and Evolved Universal Terrestrial Radio Access Network (EUTRAN); Overall description”, V11.2.0, Jun 2012.

[6] A. Barbieri, A. Damnjanovic, T. F. Ji, J. Montojo, Y. B. Wei, D. Malladi, et al., "LTE Femtocells: System Design and Performance Analysis,"
IEEE Journal on Selected Areas in Communications, vol. 30, pp. 586594, Apr 2012.

[7] S. Yoon and J. Cho, "Impact of Femtocell Deployment on Existing Macrocells," IEICE Transactions on Communications, vol. E95B, pp. 1730-1737, May 2012.

[8] C. Shun-Ping and C. Yu-Wei, "Performance Analysis of Call Admission Control in SFR-Based LTE Systems," IEEE Communications Letters, vol. 16, pp. 1014-1017, 2012.

[9] E. Pateromichelakis, M. Shariat, A. Quddus, and R. Tafazolli, "On the Evolution of Multi-Cell Scheduling in 3GPP LTE / LTE-A," IEEE Communications Surveys \& Tutorials, vol. PP, pp. 1-17, 2012.

[10] D. Lopez-Perez, X. L. Chu, and J. Zhang, "Dynamic Downlink Frequency and Power Allocation in OFDMA Cellular Networks," IEEE Transactions on Communications, vol. 60, pp. 2904-2914, Oct 2012.

[11] 3GPP, TSG TS 36.423, "X2 Application Protocol (X2AP)", V11.1.0, Jun 2012.

[12] 3GPP, TSG TR 36.921, "FDD Home eNB Radio Frequency (RF) requirements analysis," V10.0.0, Apr 2011.

[13] 3GPP, TSG TS 36.214, "Evolved Universal Terrestrial Radio Access (EUTRA); Physical layer; Measurements," V10.1.0, Mar 2011.

[14] 3GPP, TSG TS 36.211, "Physical channels and modulation," V10.5.0, Jun 2012.

[15] S. Deb, P. Monogioudis, J. Miernik, and J. P. Seymour, "Algorithms for Enhanced Inter-Cell Interference Coordination (eICIC) in LTE HetNets," IEEE/ACM Transactions on Networking, vol. PP, pp. 1-1, 2013.

[16] Ghosh, Arunabha, et al. "Fundamentals of LTE", Pearson Education, 2010.

[17] E. Dahlman, S. Parkvall, and J. Skold, 4G: "LTE/LTE-Advanced for Mobile Broadband: LTE/LTE-Advanced for Mobile Broadband:" Academic Press, 2011.

[18] C. Mehlführer, J. Colom Colom Ikuno, M. Šimko, S. Schwarz, M. Wrulich, and M. Rupp, "The Vienna LTE simulators-Enabling reproducibility in wireless communications research," EURASIP Journal on Advances in Signal Processing, vol. 2011, pp. 1-14, 2011.

[19] J. C. Ikuno, S. Pendl, M. Simko, and M. Rupp, "Accurate SINR estimation model for system level simulation of LTE networks," IEEE International Conference on Communications (ICC), pp. 1471-1475, 2012

[20] 3GPP, TSG TS 36.942, "Radio Frequency (RF) system scenarios," V11.0.0, Sep 2012. 